\title{
The most general $\lambda$-deformation of CFTs and integrability
}

\author{
George Georgiou and Konstantinos Sfetsos \\ Department of Nuclear and Particle Physics, Faculty of Physics, \\ National and Kapodistrian University of Athens, \\ Athens 15784, Greece \\ E-mail: georgiou@inp.demokritos.gr, ksfetsos@phys.uoa.gr
}

ABSTRACT: We show that the CFT with symmetry group $G_{k_{1}} \times G_{k_{2}} \times \cdots \times G_{k_{n}}$ consisting of WZW models based on the same group $G$, but at arbitrary integer levels, admits an integrable deformation depending on $2(n-1)$ continuous parameters. We derive the all-loop effective action of the deformed theory and prove integrability. We also calculate the exact in the deformation parameters RG flow equations which can be put in a particularly simple compact form. This allows a full determination and classification of the fixed points of the RG flow, in particular of those that are IR stable. The models under consideration provide concrete realizations of integrable flows between CFTs. We also consider non-Abelian T-duality type limits.

KEywords: Field Theories in Lower Dimensions, Integrable Field Theories, Sigma Models, Bosonic Strings

ARXIV EPRINT: 1812.04033 


\section{Contents}

1 Introduction 1

2 Generalities: Lagrangian and equations of motion 3

3 Integrable deformations $\quad 6$

$\begin{array}{lll}3.1 & \text { A truncation of our models } & 6\end{array}$

$\begin{array}{ll}3.2 & \text { Proof of integrability }\end{array}$

4 RG flow equations and fixed points $\quad 11$

4.1 The RG flow equations 11

$\begin{array}{lll}4.2 & \text { Fixed points } & 13\end{array}$

$\begin{array}{ll}4.3 \text { The stability matrix } & 13\end{array}$

$\begin{array}{lll}\text { 4.3.1 IR stable fixed points } & 14\end{array}$

5 The non-Abelian T-duality limit $\quad 15$

6 Discussion and future directions 16

\section{Introduction}

Integrability is one of the key features for obtaining exact results in quantum field theory (QFT). The most well-known example where integrability was greatly exploited is that of the maximally supersymmetric gauge theory in four dimensions, that is $\mathcal{N}=4 \mathrm{SYM}$. Thanks to the AdS/CFT correspondence [1] the dynamics of $\mathcal{N}=4$ SYM can be translated to those of an integrable two-dimensional non-linear $\sigma$-model evading thus certain no-go theorems which forbid integrable theories in more than two dimensions. A variety of integrability-based techniques, from the asymptotic Bethe ansatz [2] to the thermodynamic Bethe ansatz [3] and the Y-system [4], were employed in order to determine the planar anomalous dimensions of gauge invariant operators for all values of the't Hooft coupling $\lambda=g_{Y M}^{2} N$. For further developments on integrability and the AdS/CFT correspondence see [5] and references therein.

It is an intriguing endeavour to formulate new examples of gauge/gravity dualities where although supersymmetry may be broken the integrability of the theories involved will be maintained. The comments above make it clear that one starting point could be new integrable two-dimensional field theories which can serve as the seed for such constructions. One such direction which has attracted attention lately is the $\lambda$-deformed models of [6], as well as generalisations in many directions [7-10].

The aforementioned constructions provide an effective and rather effortless method for obtaining exact results in a general class of two-dimensional QFTs. The starting point of 
the construction is certain conformal field theories (CFTs) of the WZW type perturbed by current bilinear operators with the currents belonging either to the same and/or different groups. The essence of the method relies on the construction of the corresponding all-loop effective actions for the deformed theories by a gauging procedure [6-10]. These effective actions possess non-perturbative symmetries in the space of couplings [9-12]. ${ }^{1}$

Combining low-order perturbation theory with the manifestation of the aforementioned non-perturbative symmetries at the level of correlators one is able to derive exact expressions for them. This program was initiated and implemented in a series of papers in which exact expressions were obtained for many observables. In particular, when gravitational techniques were also implemented the exact in the deformation parameters $\beta$-functions were found $[9,11,12,14]$, with expressions for the most general case of anisotropic $\lambda$ deformations and different WZW levels derived in [15]. ${ }^{2}$ In addition, anomalous dimensions of current [20] and primary operators [21,22], as well as three-point correlators involving currents and/or primary operators [21, 22] were calculated for the models of [6-9]. Furthermore, the computation of the $C$-function of Zamolodchikov [23], exactly in the deformation parameter for the case of isotropic perturbations but to leading order in $k$, was performed in [24] and further generalized for anisotropic $\lambda$-deformations in [25].

In a parallel development of particular importance the extension to the case where the unperturbed CFT of a single WZW model is replaced by a coset CFT was considered in $[6,26-28]$. The deformed theory was found to be integrable when the coset is chosen to be a symmetric space [26]. Subsequently, the corresponding analysis for the case of supergroups was considered in [27]. Although integrability has not been an essential ingredient in the computation of the $\beta$-functions and of the operators anomalous dimensions, in the case of isotropic deformations the above models have been demonstrated to be integrable $[6,8,9,26-28]$. Note that for the special case of the isotropic deformation based on the $\mathrm{SU}(2)$ group the model has been initially proven to be integrable in [29]. Furthermore, integrability was shown to persist in some other cases with more deformation parameters [30, 31]. In addition, certain deformed models of low dimensionality have been embedded to type-IIA or type-IIB supergravity [32-35].

An interesting relation between $\lambda$-deformations and $\eta$-deformations for group and coset spaces was discussed in [31, 36-40]. In particular, the $\lambda$-deformed models are related via Poisson-Lie T-duality, which has been introduced for group spaces in [41] and extended for coset spaces in [42], and appropriate analytic continuations to the $\eta$-deformed models. The latter were introduced in [43-45] and [46-48] for group and coset spaces, respectively. Moreover, the dynamics of scalar fields in some $\lambda$-deformed geometries corresponding to coset CFTs has been discussed in [49] while the relation to Chern-Simons theories was discussed in [50, 51]. Finally, D-branes regarded as integrable boundary configurations were introduced in the context of $\lambda$-deformations in [52].

\footnotetext{
${ }^{1}$ For isotropic $\lambda$-deformations the simplest such non-perturbative symmetry was found initially in [13] using path integral arguments.

${ }^{2}$ The exact $\beta$-functions for CFTs deformed by operators bilinear in currents and for isotropic cases have been obtained in the past either by field theoretical methods (resummation of the perturbation series or the background field method) [16-19]. The results are in complete agreement.
} 
The models presented in $[9,10]$ have several virtues. They provide concrete realizations of flows between exact CFTs in which the Lagrangian of the theory is known all the way through from the UV to the IR fixed points. Integrability is preserved in the entire flow and therefore these flows are called integrable. Furthermore, the construction of [10] provides the first example in which self- and mutual-interactions between different WZW models are present already at leading order in the deformation parameters. In this work we continue this line of research and construct integrable multi-parameter deformations of CFTs whose Lagrangian and RG flow equations are known to all-orders in perturbation theory. The integrable sector will belong to the most general $\lambda$-deformed model which we construct. In this model self- as well as mutual-interactions are present.

The plan of the present paper is as follows: in section 2, we construct the all loop effective action of a general class of models whose UV Lagrangian is the sum of an arbitrary number $n$ of WZW models based on the same group $G$ but at different levels. In general our models depend on $n^{2}$ general coupling matrices. In section 3 , we consider a consistent truncation of our models that depend on $2(n-1)$ couplings, identify the non-perturbative symmetries in the space of couplings $\hat{\lambda}_{i 1}$ and $\hat{\lambda} n i$ and show that the theory is classically integrable by finding the appropriate Lax connection. In section 4 , we calculate the exact in the deformation parameters $\beta$-functions of our models which can be cast in a particularly simple and compact form. Subsequently, we determine and classify the fixed points of the RG flow in particular those that are IR stable. Finally, in section 5 we consider nonAbelian T-duality type limits in the case when the theory is integrable. In the last section we present our conclusions.

\section{Generalities: Lagrangian and equations of motion}

In this section we construct the effective actions of our model and derive the corresponding equations of motion.

Our starting point is to consider group elements $g_{i}, i=1,2, \ldots, n$ in a semi-simple group $G$ and the corresponding WZW model actions $S_{k_{i}}\left(g_{i}\right)$ at levels $k_{1}, k_{2}, \ldots, k_{n}$. We add to this the action of $n$ PCMs which are both self and mutually interacting and are constructed using $n$ group elements $\tilde{g}_{i}, i=1,2, \ldots, n$ belonging in the same group $G$. Namely, we have that

$$
S_{k_{i}, E_{i}}\left(g_{i}, \tilde{g}_{i}\right)=\sum_{i=1}^{n} S_{k_{i}}\left(g_{i}\right)-\frac{1}{\pi} \int d^{2} \sigma\left(\tilde{g}_{i}^{-1} \partial_{+} \tilde{g}_{i}\right)_{a} E_{i j}^{a b}\left(\tilde{g}_{j}^{-1} \partial_{-} \tilde{g}_{j}\right)_{b},
$$

where the $E_{i j}, i, j=1,2, \ldots, n$ are generic coupling matrices and the indices $a, b=1,2$, $\ldots, \operatorname{dim}(G)$. In the spirit of $[6,8,10]$ we gauge the global symmetry acting on the group elements as follows: $g_{i} \rightarrow \Lambda_{i}^{-1} g_{i} \Lambda_{i}$ and $\tilde{g}_{i} \rightarrow \Lambda_{i}^{-1} \tilde{g}_{i}, i=1,2, \ldots, n$. The resulting gauge invariant action reads

$$
S_{k_{i}, E_{i}}\left(g_{i}, \tilde{g}_{i}, A_{ \pm}^{(i)}\right)=\sum_{i=1}^{n} S_{k_{i}}\left(g_{i}, A_{ \pm}^{(i)}\right)-\frac{1}{\pi} \sum_{i, j=1}^{n} \int d^{2} \sigma\left(\tilde{g}_{i}^{-1} \tilde{D}_{+} \tilde{g}_{i}\right)_{a} E_{i j}^{a b}\left(\tilde{g}_{j}^{-1} \tilde{D}_{-} \tilde{g}_{j}\right)_{b}
$$


where $S_{k_{i}}\left(g_{i}, A_{ \pm}^{(i)}\right)$ is the standard gauged WZW action

$$
\begin{aligned}
S_{k_{i}}\left(g_{i}, A_{ \pm}^{(i)}\right)= & S_{k_{i}}\left(g_{i}\right)+\frac{k_{i}}{\pi} \int d^{2} \sigma \operatorname{Tr}\left(A_{-}^{(i)} \partial_{+} g_{i} g_{i}^{-1}-A_{+}^{(i)} g_{i}^{-1} \partial_{-} g_{i}\right. \\
& \left.+A_{-}^{(i)} g_{i} A_{+}^{(i)} g_{i}^{-1}-A_{-}^{(i)} A_{+}^{(i)}\right)
\end{aligned}
$$

where we have suppressed the group indices. Furthermore, the covariant derivatives were defined as $\tilde{D}_{ \pm} \tilde{g}_{i}=\left(\partial_{ \pm}-A_{ \pm}^{(i)}\right) \tilde{g}_{i}$. One may now fix the gauge in (2.2) choosing $\tilde{g}_{i}=\nVdash$ to arrive at the following action

$$
\begin{aligned}
S_{k_{i}, \lambda_{i j}}\left(g_{i}, A_{ \pm}^{(i)}\right)= & \sum_{i=1}^{n} S_{k_{i}}\left(g_{i}\right)+\frac{k_{i}}{\pi} \int d^{2} \sigma \operatorname{Tr}\left(A_{-}^{(i)} \partial_{+} g_{i} g_{i}^{-1}-A_{+}^{(i)} g_{i}^{-1} \partial_{-} g_{i}\right. \\
& \left.+A_{-}^{(i)} g_{i} A_{+}^{(i)} g_{i}^{-1}\right)-\frac{1}{\pi} \sum_{i, j=1}^{n} \int d^{2} \sigma \sqrt{k_{i} k_{j}} A_{+}^{(i)} \lambda_{i j}^{-1} A_{-}^{(j)}
\end{aligned}
$$

where we have redefined the coupling matrices appearing in the PCM models as ${ }^{3}$

$$
\sqrt{k_{i} k_{j}} \lambda_{i j}^{-1}=E_{i j}+k_{i} \delta_{i j}
$$

In order to obtain the $\sigma$-model and to show integrability in the next section we should integrate out the gauge fields. These are not dynamical and appear only quadratically in the action. We cast the corresponding equations of motion into the following particularly convenient form

$$
g_{i}^{-1} D_{-} g_{i}=\left(\delta_{i j}-\lambda_{i j}^{-1} \sqrt{\frac{k_{j}}{k_{i}}}\right) A_{-}^{(j)}, \quad D_{+} g_{i} g_{i}^{-1}=-\left(\delta_{i j}-\lambda_{j i}^{-T} \sqrt{\frac{k_{j}}{k_{i}}}\right) A_{+}^{(j)},
$$

where as usual we have defined $D_{ \pm} g_{i}=\partial_{ \pm} g_{i}-\left[A_{ \pm}^{(i)}, g_{i}\right]$ and where we note that the transpose in $\lambda_{i j}^{-T}$, as well as the inverses refer only to the suppressed group indices and not on the space of couplings with indices $i, j$.

Varying with respect to the group elements one obtains

$$
\begin{aligned}
D_{-}\left(D_{+} g_{i} g_{i}^{-1}\right) & =F_{+-}^{(i)} \Longleftrightarrow D_{+}\left(g_{i}^{-1} D_{-} g_{i}\right)=F_{+-}^{(i)} \\
F_{+-}^{(i)} & =\partial_{+} A_{-}^{(i)}-\partial_{-} A_{+}^{(i)}-\left[A_{+}^{(i)}, A_{-}^{(i)}\right] .
\end{aligned}
$$

To proceed we find it convenient to rescale the gauge fields and define

$$
\tilde{A}_{ \pm}^{(i)}=\sqrt{k_{i}} A_{ \pm}^{(i)}
$$

\footnotetext{
${ }^{3}$ To compare with the corresponding actions in [10] (see eqs. (2.1) and (2.4)) corresponding to two WZW models self- and mutually interacting we have

$$
\begin{array}{llll}
E_{11}=E_{1}, & E_{22}=E_{4}, & E_{12}=E_{2}, & E_{21}=E_{3}, \\
\lambda_{11}=\sqrt{\frac{k_{1}}{k_{2}} \lambda_{4},} & \lambda_{22}=\sqrt{\frac{k_{2}}{k_{1}}} \lambda_{3}, & \lambda_{12}=\lambda_{2}, & \lambda_{21}=\lambda_{1} .
\end{array}
$$
}


Using them and substituting (2.6) in (2.7) we obtain after some algebra that

$$
\begin{aligned}
& \lambda_{i j}^{-1} \partial_{+} \tilde{A}_{-}^{(j)}-\partial_{-} \tilde{A}_{+}^{(i)}=\frac{1}{\sqrt{k_{i}}}\left[\tilde{A}_{+}^{(i)}, \lambda_{i j}^{-1} \tilde{A}_{-}^{(j)}\right], \\
& \partial_{+} \tilde{A}_{-}^{(i)}-\lambda_{j i}^{-T} \partial_{-} \tilde{A}_{+}^{(j)}=\frac{1}{\sqrt{k_{i}}}\left[\lambda_{j i}^{-T} \tilde{A}_{+}^{(j)}, \tilde{A}_{-}^{(i)}\right] .
\end{aligned}
$$

To present the $\sigma$-model action we introduce representation Hermitian matrices $t_{a}$ and define the currents and primary field to be

$$
J_{+}^{a}=-i \operatorname{Tr}\left(t^{a} \partial_{+} g g^{-1}\right), \quad J_{-}^{a}=-i \operatorname{Tr}\left(t^{a} g^{-1} \partial_{-} g\right), \quad D^{a b}=\operatorname{Tr}\left(t^{a} g t^{b} g^{-1}\right) .
$$

These will be computed for the particular group elements $g_{i}$ in which case an extra index $i$ will be inserted in the above quantities. We also define the diagonal in the coupling space matrix

$$
\mathcal{D}_{i j}=D_{i} \delta_{i j}
$$

Then we may solve for the redefined gauge fields using (2.6) to obtain

$$
\tilde{A}_{+}^{(i)}=\left(\lambda^{-T}-\mathcal{D}\right)_{i j}^{-1} \sqrt{k_{j}} J_{j+}, \quad \tilde{A}_{-}^{(i)}=-\left(\lambda^{-1}-\mathcal{D}^{T}\right)_{i j}^{-1} \sqrt{k_{j}} J_{j-} .
$$

Note that the entries of the matrices $\lambda^{-T}-\mathcal{D}$ and $\lambda^{-1}-\mathcal{D}^{T}$ are themselves matrices in the group $G$. Thus, their inversion is to be understood as an inversion in the space of different models keeping in mind that their entries are non-commutative objects.

Finally, substituting the values for the gauge fields in the action (2.4) we obtain the following $\sigma$-model

$$
S_{k_{i}, \lambda}\left(g_{i}\right)=\sum_{i=1}^{n} S_{k_{i}}\left(g_{i}\right)+\frac{1}{\pi} \sum_{i, j=1}^{n} \int d^{2} \sigma \sqrt{k_{i}} J_{i+}\left(\lambda^{-1}-\mathcal{D}^{T}\right)_{i j}^{-1} \sqrt{k_{j}} J_{-j} .
$$

This is a particularly compact form resembling the single $\lambda$-deformed model of [6]. The above action encompasses all previous ones for $\lambda$-deformed models. Furthermore it is the most general action that can be construct using the same group $G$ for all couplings. A further generalization in which a different group is associated to each WZW model can be constructed straightforwardly.

For small entries in the matrices $\lambda_{i j}$ the action becomes

$$
S_{k_{i}, \lambda}\left(g_{i}\right)=\sum_{i=1}^{n} S_{k_{i}}\left(g_{i}\right)+\frac{1}{\pi} \sum_{i, j=1}^{n} \int d^{2} \sigma \sqrt{k_{i}} J_{i+}\left(\lambda^{-1}\right)_{i j}^{-1} \sqrt{k_{j}} J_{-j}+\cdots
$$

Note that $\left(\lambda^{-1}\right)_{i j}^{-1} \neq \lambda_{i j}$ since the inverse in $\lambda^{-1}$ is taken in the group space, and the dots denote subleading terms in the small $\lambda$-expansion. 
Finally, note that in integrating out the gauge field a dilaton field is generated. The value of the dilaton is proportional to the logarithm of the determinant of the square matrix $\lambda^{-1}-\mathcal{D}^{T}$ inverted in (2.12). Note that the dimensionally of this matrix is $n \operatorname{dim} G$.

In the next section the inversion of the above matrices in the coupling space will be done explicitly for the case of integrable models.

\section{Integrable deformations}

In this section we will attempt to answer the following question. For which choices of the matrix $E_{i j}^{a b}$ appearing in (2.1) is the theory described by (2.13) integrable? To the best of our knowledge we are lacking a general answer to this question even for the case of a single group $G$. Nevertheless, it has been proven that there are several cases where these theories are integrable for specific choices of the couplings matrices. Among the single $\lambda$-deformations the first one is the case of the isotropic $\lambda$-deformation, that is when $\lambda_{a b}=\lambda \delta_{a b}[6]$, a second one is the case where the matrix $E$ is of the form $E=\frac{1}{t}(1-\eta R)^{-1}$, where the matrix $R$ satisfies the modified Yang-Baxter equation [44, 45]. Another case is that where instead of the group $G$ one has a coset with the coset being a symmetric space $[6,26] .{ }^{4}$ Furthermore, integrability has been shown for the models of $[8,9]$ and [10] representing particular cases of self- and mutual-interactions of current algebras based on WZW models.

\subsection{A truncation of our models}

In what follows, we will show that the theory (2.13) is integrable in the case where the matrices $\lambda_{i j}$ is of the following form

$$
\begin{aligned}
& \lambda_{i 1}^{-1} \neq 0, \quad i=1,2, \ldots, n-1, \quad \lambda_{n j}^{-1} \neq 0, \quad j=2,3, \ldots, n, \\
& \lambda_{i j}^{-1}=0 \text { for all other entries } .
\end{aligned}
$$

Hence, the corresponding coupling matrices reads

$$
\lambda_{i j}^{-1}=\left(\begin{array}{cccc}
\lambda_{11}^{-1} & 0 & \cdots & 0 \\
\lambda_{21}^{-1} & 0 & \cdots & 0 \\
\vdots & \vdots & \ddots & \vdots \\
\lambda_{(n-1) 1}^{-1} & 0 & \cdots & 0 \\
0 & \lambda_{n 2}^{-1} & \cdots & \lambda_{n n}^{-1}
\end{array}\right) .
$$

As a result it will be shown that one obtains an integrable deformation of the $G_{k_{1}} \times G_{k_{2}} \times$ $\cdots \times G_{k_{n}}$ depending on $2(n-1)$ parameters. This provides a partial answer to the question posed in the beginning of this section. We stress that by turning on just one more coupling, in addition to those appearing in (3.2), it will generate other couplings through quantum corrections. Then, the theory will most likely cease to be integrable. However, we have no proof that (3.2) exhausts all integrable cases among our general class of models.

\footnotetext{
${ }^{4}$ For the case of the anisotropic $\lambda$-deformed SU(2) model integrability was shown in [30].
} 
Turning to the general action (2.13), after using (3.1) and explicitly inverting the relevant matrix in the coupling space we find that

$$
\begin{aligned}
S_{k_{i}, \lambda}\left(g_{i}\right)= & \sum_{i=1}^{n} S_{k_{i}}\left(g_{i}\right)+\frac{k_{1}}{\pi} \int d^{2} \sigma J_{1+}\left(\lambda_{11}^{-1}-D_{1}^{T}\right)^{-1} J_{1-} \\
& +\frac{k_{n}}{\pi} \int d^{2} \sigma J_{n+}\left(\lambda_{n n}^{-1}-D_{n}^{T}\right)^{-1} J_{n-}-\frac{1}{\pi} \sum_{i=2}^{n-1} k_{i} \int d^{2} \sigma J_{+i} D_{i} J_{i-} \\
& +\frac{1}{\pi} \sum_{i=2}^{n-1} \sqrt{k_{1} k_{i}} \int d^{2} \sigma J_{i+} D_{i} \lambda_{i 1}^{-1}\left(\lambda_{11}^{-1}-D_{1}^{T}\right)^{-1} J_{1-} \\
& +\frac{1}{\pi} \sum_{i=2}^{n-1} \sqrt{k_{n} k_{i}} \int d^{2} \sigma J_{n+}\left(\lambda_{n n}^{-1}-D_{n}^{T}\right)^{-1} \lambda_{n i}^{-1} D_{i} J_{i-} \\
& -\frac{1}{\pi} \sum_{i=2}^{n-1} \sqrt{k_{n} k_{1}} \int d^{2} \sigma J_{n+}\left(\lambda_{n n}^{-1}-D_{n}^{T}\right)^{-1} \lambda_{n i}^{-1} D_{i} \lambda_{i 1}^{-1}\left(\lambda_{11}^{-1}-D_{1}^{T}\right)^{-1} J_{1-} .
\end{aligned}
$$

From this point on we will focus for simplicity on the case where the couplings are taken isotropic in the group space, that is

$$
\lambda_{i j}^{a b}=\delta^{a b} \lambda_{i j}
$$

One now encounters the following problem. By combining $S_{k_{i}}\left(g_{i}\right), i=2, \ldots, n-1$ with the last term in the second line of (3.3) we obtain a sum of WZW model actions $S_{-k_{i}}\left(g_{i}^{-1}\right), i=$ $2, \ldots, n-1$ with negative signature (this is also true for the PCM part of the action (2.1) before the gauging procedure is performed). As was pointed out in the case of two interacting WZW models in [10] to remedy this situation one can perform the following redefinitions of the couplings and analytic continuation in the following specified order. First define the hatted couplings

$$
\hat{\lambda}_{i 1}=\lambda_{11} \lambda_{i 1}^{-1} \sqrt{\frac{k_{1}}{k_{i}}}, \quad \hat{\lambda}_{n i}=\lambda_{n n} \lambda_{n i}^{-1} \sqrt{\frac{k_{n}}{k_{i}}}, \quad i=2,3, \ldots, n-1
$$

and then flip the signs of levels and invert the corresponding group elements as

$$
k_{i} \rightarrow-k_{i}, \quad g_{i} \rightarrow g_{i}^{-1}, \quad i=2,3, \ldots, n-1 .
$$

Then the action (3.3) becomes

$$
\begin{aligned}
S_{k_{i}, \lambda}\left(g_{i}\right)= & \sum_{i=1}^{n} S_{k_{i}}\left(g_{i}\right) \\
& +\frac{k_{1}}{\pi} \int d^{2} \sigma J_{1+}\left(\lambda_{11}^{-1} \nVdash-D_{1}^{T}\right)^{-1} J_{1-}+\frac{k_{n}}{\pi} \int d^{2} \sigma \quad J_{n+}\left(\lambda_{n n}^{-1} \nVdash-D_{n}^{T}\right)^{-1} J_{n-} \\
& +\frac{1}{\pi} \sum_{i=2}^{n-1} k_{i} \int d^{2} \sigma \lambda_{11}^{-1} \hat{\lambda}_{i 1} J_{i+}\left(\lambda_{11}^{-1} \nVdash-D_{1}^{T}\right)^{-1} J_{1-} \\
& +\frac{1}{\pi} \sum_{i=2}^{n-1} k_{i} \int d^{2} \sigma \lambda_{n n}^{-1} \hat{\lambda}_{n i} J_{n+}\left(\lambda_{n n}^{-1} \nVdash-D_{n}^{T}\right)^{-1} J_{i-} \\
& +\frac{1}{\pi} \sum_{i=2}^{n-1} k_{i} \int d^{2} \sigma \lambda_{11}^{-1} \lambda_{n n}^{-1} \hat{\lambda}_{n i} \hat{\lambda}_{i 1} J_{n+}\left(\lambda_{n n}^{-1} \nVdash-D_{n}^{T}\right)^{-1} D_{i}^{T}\left(\lambda_{11}^{-1} \nVdash-D_{1}^{T}\right)^{-1} J_{1-} .
\end{aligned}
$$


This is the final expression for the all-loop effective action of our $\sigma$-model. In what follows we will prove that it is indeed classically integrable.

Before that, we comment on the constraints imposed by demanding a non-singular $\sigma$-model of Euclidean signature. In order to avoid singularities the couplings $\lambda_{11}$ and $\lambda_{n n}$ should be such that $\left|\lambda_{11}\right|,\left|\lambda_{n n}\right|<1$, the reason being that the matrix $D$ is orthogonal and therefore its eigenvalues lie in the unit circle. In addition, similarly to [10], it can be shown that the signature of (3.7) is Euclidean provided that the couplings lie within the ellipsoids defined by

$$
\Delta_{1}=1-\lambda_{11}^{2}-\sum_{i=2}^{n-1} \frac{k_{i}}{k_{1}} \hat{\lambda}_{i 1}^{2}>0 \quad \text { and } \quad \Delta_{2}=1-\lambda_{n n}^{2}-\sum_{i=2}^{n-1} \frac{k_{i}}{k_{n}} \hat{\lambda}_{n i}^{2}>0 .
$$

It is straightforward to show that the action (3.7) is independently invariant under the following two non-perturbative symmetry operations, as well as under their combination

$$
\begin{array}{ll}
(\mathrm{A}): & \lambda_{11} \rightarrow \frac{1}{\lambda_{11}}, \quad \hat{\lambda}_{i 1} \rightarrow \frac{\hat{\lambda}_{i 1}}{\lambda_{11}}, \quad k_{1} \rightarrow-k_{1}, \quad g_{1} \rightarrow g_{1}^{-1}, \quad i=2, \ldots, n-1 \\
(\mathrm{~B}): & \lambda_{n n} \rightarrow \frac{1}{\lambda_{n n}}, \quad \hat{\lambda}_{n i} \rightarrow \frac{\hat{\lambda}_{n i}}{\lambda_{n n}}, \quad k_{n} \rightarrow-k_{n}, \quad g_{n} \rightarrow g_{n}^{-1}, \quad i=2, \ldots, n-1 .
\end{array}
$$

This is a generalization of the corresponding symmetry found in [10].

Expanding (3.7) and keeping the linear terms in the couplings one obtains

$$
\begin{aligned}
S_{k_{i}, \lambda}\left(g_{i}\right)= & \sum_{i=1}^{n} S_{k_{i}}\left(g_{i}\right)+\frac{1}{\pi} \int d^{2} \sigma\left(k_{1} \lambda_{11} J_{1+}^{a} J_{1-}^{a}+k_{n} \lambda_{n n} J_{n+}^{a} J_{n-}^{a}\right) \\
& +\frac{1}{\pi} \sum_{i=2}^{n-1} k_{i} \int d^{2} \sigma\left(\hat{\lambda}_{i 1} J_{i+}^{a} J_{1-}^{a}+\hat{\lambda}_{n i} J_{n+}^{a} J_{i-}^{a}\right)+\mathcal{O}\left(\lambda^{2}\right) .
\end{aligned}
$$

Notice the last line of (3.7) has disappeared from the small coupling expansion since it is quadratic in the $\lambda$ 's. This term, as well as the full action of (3.7) is generated when quantum corrections at arbitrary order in perturbation theory are taken into account.

Note that there is the following interesting truncation of (3.7) which dramatically further simplifies it. If we let $\lambda_{11}=\lambda_{n n}=0$ then we obtain

$$
\begin{aligned}
S_{k_{i}, \lambda}\left(g_{i}\right)= & S_{k_{1}}\left(g_{1}\right)+S_{k_{n}}\left(g_{n}\right)+\sum_{i=2}^{n-1} S_{k_{i}}\left(g_{i}\right) \\
& +\frac{1}{\pi} \sum_{i=2}^{n-1} k_{i} \int d^{2} \sigma\left(\hat{\lambda}_{i 1} J_{i+} J_{1-}+\hat{\lambda}_{n i} J_{n+} J_{i-}+\hat{\lambda}_{n i} \hat{\lambda}_{i 1} J_{n+} D_{i}^{T} J_{1-}\right) .
\end{aligned}
$$

This effective action is exact in $\lambda$ and is at most quadratic in the couplings and represents only mutual interactions between the various WZW models as depicted. It becomes linear if we make the further truncation $\hat{\lambda}_{n 1}=0$ or $\hat{\lambda}_{i 1}=0$. 


\subsection{Proof of integrability}

To prove that (3.7) is integrable we write the equations of motion (2.9) for the choice of couplings appearing in (3.1). The first equation of (2.9) can be decomposed to

$$
\begin{aligned}
& \lambda_{i 1}^{-1} \partial_{+} \tilde{A}_{-}^{(1)}-\partial_{-} \tilde{A}_{+}^{(i)}=\frac{1}{\sqrt{k_{i}}}\left[\tilde{A}_{+}^{(i)}, \lambda_{i 1}^{-1} \tilde{A}_{-}^{(1)}\right], \quad i=1,2, \ldots, n-1, \\
& \lambda_{n i}^{-1} \partial_{+} \tilde{A}_{-}^{(i)}-\partial_{-} \tilde{A}_{+}^{(n)}=\frac{1}{\sqrt{k_{n}}}\left[\tilde{A}_{+}^{(n)}, \lambda_{n i}^{-1} \tilde{A}_{-}^{(i)}\right] .
\end{aligned}
$$

while the second one to

$$
\begin{aligned}
& \partial_{+} \tilde{A}_{-}^{(1)}-\lambda_{i 1}^{-1} \partial_{-} \tilde{A}_{+}^{(i)}=\frac{1}{\sqrt{k_{1}}}\left[\lambda_{i 1}^{-1} \tilde{A}_{+}^{(i)}, \tilde{A}_{-}^{(1)}\right], \\
& \partial_{+} \tilde{A}_{-}^{(i)}-\lambda_{n i}^{-1} \partial_{-} \tilde{A}_{+}^{(n)}=\frac{1}{\sqrt{k_{i}}}\left[\lambda_{n i}^{-1} \tilde{A}_{+}^{(n)}, \tilde{A}_{-}^{(i)}\right], \quad i=2,3, \ldots, n .
\end{aligned}
$$

The first line equations in (3.12) can be combined with the equation in the first line in (3.13) to form a system depending only on the $\lambda_{i 1}^{-1}$ 's but not on $\lambda_{n i}^{-1}$ which furthermore can be used to solve for the $n$ derivatives of the gauge fields, $\partial_{+} \tilde{A}_{-}^{(1)}$ and $\partial_{-} \tilde{A}_{+}^{(i)}, i=1, \ldots, n-1$. Similarly, we may use the equation in second line of (3.12) and the equations in the second line in (3.13) to form a system of equations that depend only on the $\lambda_{n i}^{-1}$ 's, but not on the $\lambda_{i 1}^{-1}$ 's. This allows to solve for the $n$ derivatives of the gauge fields $\partial_{-} \tilde{A}_{+}^{(n)}$ and $\partial_{+} \tilde{A}_{-}^{(i)}$, $i=2, \ldots, n$. The two aforementioned sets of equations are decoupled. We stress that even though in these systems the couplings $\lambda_{i 1}^{-1}$ completely disentangled from the $\lambda_{n i}^{-1}$ 's this is not the case in the effective action (3.7) where the last term involves both sets of couplings. The reason is that the various gauge fields depend on all group elements $g_{i}, i=1,2, \ldots, n$.

Our strategy will be to determine a Lax pair for each set of equations and then show that the charges obtained from the first Lax pair are in involution with those obtained from the second one, thus proving that the theory is integrable. To this end we define the rescaled anti-commutators

$$
\left[\tilde{A}_{+}^{(i)}, \tilde{A}_{-}^{(j)}\right]_{* L}=\frac{1}{\sqrt{k_{i}}}\left[\tilde{A}_{+}^{(i)}, \tilde{A}_{-}^{(j)}\right], \quad\left[A_{+}^{(i)}, A_{-}^{(j)}\right]_{* R}=\frac{1}{\sqrt{k_{j}}}\left[A_{+}^{(i)}, A_{-}^{(j)}\right] .
$$

In terms of these redefined quantities the solution to the first set of equations reads

$$
\begin{aligned}
& \partial_{+} \tilde{A}_{-}^{(1)}=\frac{1}{d} \sum_{i=1}^{n-1}\left(\mu_{i 1}-\lambda_{i 1}^{-1}\right) \lambda_{i 1}^{-1}\left[\tilde{A}_{+}^{(i)}, \tilde{A}_{-}^{(1)}\right]_{* L}, \quad \mu_{i 1}=\sqrt{\frac{k_{i}}{k_{1}}}, \quad d=1-\sum_{i=1}^{n-1} \lambda_{i 1}^{-2}, \\
& \partial_{-} \tilde{A}_{+}^{(i)}=\frac{\lambda_{i 1}^{-1}}{d}\left(-d\left[\tilde{A}_{+}^{(i)}, \tilde{A}_{-}^{(1)}\right]_{* L}+\sum_{j=1}^{n-1}\left(\mu_{j 1}-\lambda_{j 1}^{-1}\right) \lambda_{j 1}^{-1}\left[\tilde{A}_{+}^{(j)}, \tilde{A}_{-}^{(1)}\right]_{* L}\right) .
\end{aligned}
$$

These imply the existence of a Lax pair which is constructed by first assuming that this is of the form

$$
\mathcal{L}_{+}^{(1)}=\sum_{i=1}^{n-1} c_{+}^{(i)}(z) \tilde{A}_{+}^{(i)}, \quad \mathcal{L}_{-}^{(1)}=z \tilde{A}_{-}^{(1)}
$$


where $c_{+}^{(i)}$ are constants depending on the matrix of the couplings, on the WZW levels as well as on the spectral parameter. After substituting into the Lax equation

$$
\partial_{+} \mathcal{L}_{-}^{(1)}-\partial_{-} \mathcal{L}_{+}^{(1)}-\left[\mathcal{L}_{+}^{(1)}, \mathcal{L}_{-}^{(1)}\right]=0
$$

and using (3.15) one obtains a system $n$ equations which arises by equating to zero the coefficients of $\left[\tilde{A}_{+}^{(i)}, \tilde{A}_{-}^{(1)}\right]_{* L}$.

$$
\frac{z}{d}-\frac{1}{d} \sum_{j=1}^{n-1}\left(c_{+}^{(j)} \lambda_{j 1}^{-1}\right)+\frac{\left(\lambda_{i 1}^{-1}-z \sqrt{k_{i}}\right)}{\lambda_{i 1}^{-1}\left(\mu_{i 1}-\lambda_{i 1}^{-1}\right)} c_{+}^{(i)}=0 .
$$

This system can be solved for unknowns $c_{+}^{(i)}, i=1, \ldots, n-1$ and its solution reads

$$
c_{+}^{(i)}=\frac{\lambda_{i 1}^{-1}\left(\lambda_{i 1}^{-1}-\mu_{i 1}\right)}{\left(\lambda_{i 1}^{-1}-z \sqrt{k_{i}}\right)} \frac{z}{d+d_{1}}, \quad i=1,2, \ldots, n-1, \quad d_{1}=\sum_{j=1}^{n-1} \frac{\lambda_{j 1}^{-2}\left(\lambda_{j 1}^{-1}-\mu_{j 1}\right)}{\lambda_{j 1}^{-1}-z \sqrt{k_{j}}} .
$$

In conclusion we have shown that the equations of motion (3.15) imply the existence of a Lax pair from which an infinite tower of conserved charges can be calculated.

In a similar manner, the second system of equations can be solved for the derivatives of the gauge fields as

$$
\begin{aligned}
\partial_{-} \tilde{A}_{+}^{(n)} & =\frac{1}{\hat{d}} \sum_{i=2}^{n}\left(\mu_{n i}-\lambda_{n i}^{-1}\right) \lambda_{n i}^{-1}\left[\tilde{A}_{+}^{(n)}, \tilde{A}_{-}^{(i)}\right]_{* R}, \quad \mu_{n i}=\sqrt{\frac{k_{i}}{k_{n}}}, \quad \hat{d}=1-\sum_{i=2}^{n} \lambda_{n i}^{-2}, \\
\partial_{+} \tilde{A}_{-}^{(i)} & =\frac{\lambda_{n i}^{-1}}{\hat{d}}\left(-\hat{d}\left[\tilde{A}_{+}^{(n)}, \tilde{A}_{-}^{(i)}\right]_{* R}+\sum_{j=2}^{n}\left(\mu_{n j}-\lambda_{n j}^{-1}\right) \lambda_{n j}^{-1}\left[\tilde{A}_{+}^{(n)}, \tilde{A}_{-}^{(j)}\right]_{* R}\right) .
\end{aligned}
$$

The corresponding Lax pair will be of the form

$$
\mathcal{L}_{-}^{(2)}=\sum_{i=2}^{n} c_{-}^{(i)} \tilde{A}_{-}^{(i)}, \quad \mathcal{L}_{+}^{(2)}=z \tilde{A}_{+}^{(n)}
$$

The flatness of this Lax pair is guaranteed when

$$
c_{-}^{(i)}=\frac{\lambda_{n i}^{-1}\left(\lambda_{n i}^{-1}-\mu_{n i}\right)}{\left(\lambda_{n i}^{-1}-z \sqrt{k_{i}}\right)} \frac{z}{\hat{d}+\hat{d}_{1}}, \quad i=2, \ldots, n, \quad \hat{d}_{1}=\sum_{j=2}^{n} \frac{\lambda_{n j}^{-2}\left(\lambda_{n j}^{-1}-\mu_{n j}\right)}{\lambda_{n j}^{-1}-z \sqrt{k_{j}}} .
$$

The conserved charges obtained from (3.16) and (3.21) are in involution. This is so because any the gauge fields appearing in (3.16) have zero Poisson bracket with any of the gauge fields appearing in (3.21). To see this one may define, similarly to what was first done in the Hamiltonian treatment of gauged WZW models in [53], the following dressed currents $j_{ \pm}^{(i)}$ which satisfy the usual Kac-Moody algebra at level $k_{i}$

$$
\jmath_{+}^{(i)}=D_{+} g_{i} g_{i}^{-1}+A_{+}^{(i)}-A_{-}^{(i)}, \quad \jmath_{-}^{(i)}=-g_{i}^{-1} D_{-} g_{i}-A_{+}^{(i)}+A_{-}^{(i)}
$$


Using these definitions and the constraints (2.6) one obtains

$$
\begin{aligned}
\jmath_{+}^{(1)} & =\sum_{i=1}^{n-1} \sqrt{\frac{k_{i}}{k_{1}}} \lambda_{i 1}^{-T} A_{+}^{(i)}-A_{-}^{(1)}, \\
\jmath_{-}^{(i)} & =\sqrt{\frac{k_{1}}{k_{i}}} \lambda_{i 1}^{-1} A_{-}^{(1)}-A_{+}^{(i)}, \quad i=1,2, \ldots, n-1 .
\end{aligned}
$$

These can be inverted to express $A_{-}^{(1)}$ and the $A_{+}^{(i)}$ 's in terms of $\jmath_{+}^{(1)}$ and the $\jmath_{-}^{(i)}$ 's. In precisely the same way the gauge fields $A_{+}^{(n)}$ and $A_{-}^{(i)}$ can be expressed in terms of the dressed currents $\jmath_{-}^{(n)}$ and $\jmath_{+}^{(i)}$. Due to the fact that the first set of currents has zero Poisson brackets with those of the second set we conclude that $\left\{\mathcal{L}_{ \pm}^{(1)}, \mathcal{L}_{+}^{(2)}\right\}_{P B}=0$ and as a result the conserved charges obtained from the monodromy matrix involving the first Lax pair are in involution with those obtained from the monodromy matrix involving the second. This concludes the proof that the CFT deformed by the $2(n-1)$ deformation parameters $\lambda_{i 1}^{-1}, i=1,2, \ldots, n-1$ and $\lambda_{n i}^{-1}, i=2,3, \ldots, n$ is integrable.

A final comment is in order. The careful reader may have noticed that the analysis for integrability has been done using the equations of motion (3.12) and (3.13) in which the analytic continuation discussed in (3.6) has not been applied yet. However, one can easily see that the analytic continuation of (3.6) can be straightforwardly be done in the final expressions for the Lax pairs (3.16) and (3.21) once the latter are expressed in terms of the usual gauge fields $A_{ \pm}^{(i)}$ and the couplings $\hat{\lambda}_{i 1}$ and $\hat{\lambda}_{n i}$ appearing in the $\sigma$-model (3.7).

\section{RG flow equations and fixed points}

\subsection{The RG flow equations}

In this section we will first calculate the running of the couplings in the case of the integrable deformations presented in the previous section. We will need the system of RG flow equations for the running of the couplings of the anisotropic $\lambda$-model [12]

$$
\beta_{A B}=\frac{\mathrm{d} \Lambda_{A B}}{\mathrm{~d} t}=\frac{1}{2 k} \mathcal{N}(\Lambda)_{A C}{ }^{D} \mathcal{N}\left(\Lambda^{T}\right)_{B D}{ }^{C},
$$

where $t=\ln \mu^{2}, \mu$ being the energy scale and where

$$
\begin{aligned}
\mathcal{N}(\Lambda)_{A B}{ }^{c} & =\left(\Lambda_{A E} \Lambda_{B D} f_{E D F}-f_{A B E} \Lambda_{E F}\right) g^{F C}, \\
\tilde{g}_{A B} & =\left(\mathbb{I}-\Lambda \Lambda^{T}\right)_{A B}, \quad g_{A B}=\left(\mathbb{I}-\Lambda^{T} \Lambda\right)_{A B} .
\end{aligned}
$$

In what follows each of the capital indices of (4.1) will be split in two, that is $A=(i a)$, where $i, j, k=1,2, \ldots, n-1$ enumerate the different groups while $a, b, c=1, \ldots, \operatorname{dim}(G)$ denote as usual group indices. Furthermore, in order to take into account for the different levels $k_{i}$ of the WZW models, one should set $k=1$ in (4.1) and rescale the structure constants of each group as $f_{A B C}=f_{(i a)(i b)(i c)}=f_{a b c} / \sqrt{k_{i}}$. All structure constants that do not have the indices enumerating the different groups equal are set, of course to zero. 
Finally, the coupling constants matrix $\lambda_{A B}$ can be read from (3.10). It reads

$$
\Lambda_{i j}=\left(\begin{array}{cccc}
\lambda_{11} & 0 & \cdots & 0 \\
\hat{\lambda}_{21} \sqrt{\frac{k_{2}}{k_{1}}} & 0 & \cdots & 0 \\
\vdots & \vdots & \ddots & \vdots \\
\hat{\lambda}_{(n-1) 1} \sqrt{\frac{k_{n-1}}{k_{1}}} & 0 & \cdots & 0 \\
0 & \hat{\lambda}_{n 2} \sqrt{\frac{k_{2}}{k_{n}}} & \cdots & \lambda_{n n}
\end{array}\right) .
$$

Keeping in mind that the couplings of the first column in (4.3) decouple from the ones in the last row of the same equation we focus on the first column and rename $\lambda_{11}$ by $\lambda_{1}$ and similarly $\hat{\lambda}_{i 1}$ by $\lambda_{i}$, where $i=2,3, \ldots, n-1 .^{5}$ Then the non-zero components of $\mathcal{N}_{i a, i b}{ }^{j c}(\Lambda)$ and $\mathcal{N}_{1 a, 1 b}{ }^{i c}\left(\Lambda^{T}\right)$ are

$$
\begin{array}{rlrl}
\mathcal{N}_{i a, i b}{ }^{1 c}(\Lambda) & =\frac{\lambda_{i}}{\sqrt{k_{1}} \Delta}\left(\frac{k_{i}}{k_{1}} \lambda_{i}-1\right) f_{a b c}, & i=1,1, \ldots, n-1, & \\
\mathcal{N}_{1 a, i b}{ }^{1 c}(\Lambda)=\frac{\lambda_{1} \lambda_{i} \sqrt{k_{i}}}{k_{1} \Delta} f_{a b c}, & i=2,3, \ldots, n-1 \\
\mathcal{N}_{i a, j b}{ }^{1 c}(\Lambda)=\frac{\lambda_{i} \lambda_{j} \sqrt{k_{i} k_{j}}}{k_{1}^{3 / 2} \Delta} f_{a b c}, & i \neq j, \quad i, j=2,3, \cdot, n-1,
\end{array}
$$

where

$$
\Delta=1-\sum_{i=1}^{n-1} \frac{k_{i}}{k_{1}} \lambda_{i}^{2}
$$

is the same quantity, called $\Delta_{1}$ in (3.8), after the renaming of the couplings we mentioned. Furthermore,

$$
\mathcal{N}_{1 a, 1 b}{ }^{i c}\left(\Lambda^{T}\right)=\frac{\lambda_{i} \sqrt{k_{i}}}{k_{1} \Delta}\left(\lambda_{i} \Delta-1+\sum_{j=1}^{n-1} \frac{k_{j}}{k_{1}} \lambda_{j}^{3}\right) f_{a b c}, \quad i=1,2, \ldots, n-1 .
$$

Notice that $\mathcal{N}_{i a, j b}{ }^{k c}(\Lambda)=-\mathcal{N}_{j b, i a}{ }^{k c}(\Lambda)$ and $\mathcal{N}_{i a, j b}{ }^{k c}\left(\Lambda^{T}\right)=-\mathcal{N}_{j b, i a}{ }^{k c}\left(\Lambda^{T}\right)$. Using the expressions above we finally obtain for the running of the couplings the following formula

$$
\beta_{i}=-\frac{c_{G}}{2 k_{1}} \frac{\lambda_{i}\left(1-\lambda_{i}\right)\left(\lambda_{i} \Delta-Z\right)}{\Delta^{2}}, \quad i=1,2, \ldots, n-1
$$

where

$$
Z=\sum_{i=1}^{n-1} \frac{k_{i}}{k_{1}} \lambda_{i}^{2}\left(1-\lambda_{i}\right)
$$

We note that despite of the privileged rôle of $\lambda_{1}$ in the action (3.7) (see also (3.10)) the $\beta$-functions for all couplings are on equal footing and can be obtained from a single expression (4.7). This fact will allow us to fully determine the fixed points of the RGflow in the next sections. Furthermore, the $\beta$-functions in (4.7) are invariant under the non-perturbative symmetry of (3.9).

Finally, similar expressions hold for the running of the couplings $\hat{\lambda}_{n i}$. The same is true for the analysis following in this section.

\footnotetext{
${ }^{5}$ It turns out that the form of the matrix $\Lambda$ in (4.3) is preserved under the RG-flow equation (4.1).
} 


\subsection{Fixed points}

We now determine the fixed points of the RG flow equations (4.7). Each fixed point belongs to one of the following classes. Each class is characterised by three integers $\left(n_{1}, n_{2}, n_{3}\right)$, obeying the condition $n_{1}+n_{2}+n_{3}=n-1$. By $n_{1}$ we denote the number of the couplings that are set to zero, that is $\lambda_{m_{i}}=0, i=1,2, \ldots, n_{1}$. These couplings can be distributed randomly among the complete set of the $n-1$ couplings. By $n_{2}$ we denote the number of the couplings that are set to one, that is $\lambda_{p_{i}}=1, i=1,2, \ldots, n_{2}$. Finally, by $n_{3}$ we denote the number of couplings that are neither zero nor one, i.e. $\lambda_{q_{i}} \neq 0,1$, with $i=1,2, \ldots, n_{3}$. By subtracting pairwise the equations in (4.7) it is straightforward to see that all the $\lambda_{q_{i}}$ which are neither zero nor one should be equal to each other, that is $\lambda_{q_{i}}=\lambda_{*}, i=1,2, \ldots, n_{3}$. Then the quantity $\Delta$ becomes

$$
\Delta=1-\frac{\rho}{k_{1}}-\frac{\zeta}{k_{1}} \lambda_{*}^{2}, \quad \rho=\sum_{i=1}^{n_{2}} k_{p_{j}}, \quad \zeta=\sum_{i=1}^{n_{3}} k_{q_{j}} .
$$

As a result the vanishing of the bracket in (4.7) implies

$$
\lambda_{*}=\frac{k_{1}-\rho}{\zeta} .
$$

We will assume in the rest of our analysis that $n_{3} \geqslant 1$ since it turns out that this should be the case for the existence of physical IR stable fixed points.

Let us note that each of the distinct classes of fixed points characterised by $\left(n_{1}, n_{2}, n_{3}\right)$, that obey the condition $n_{1}+n_{2}+n_{3}=n-1$ has $\frac{(n-1) !}{n_{1} ! n_{2} ! n_{3} !}$ different members.

\subsection{The stability matrix}

We evaluate the stability matrix for each of the fixed points given by (4.10). This will allow us to determine the relevant and irrelevant directions of each of the fixed points and identify the fixed points which are IR stable.

Let's split the index $i=(m, a, \alpha)$ in such a way that

$$
\begin{array}{rlrl}
\lambda_{m} & =0, & m & =1,2, \ldots, n_{1}, \\
\lambda_{a} & =1, & a & =1,2, \ldots, n_{2}, \\
\lambda_{\alpha} & =\lambda_{*}=\frac{k_{1}-\rho}{\zeta}, \quad \alpha & =1,2, \ldots, n_{3},
\end{array}
$$

Then at the FPs we have the following relations

$$
\Delta=\frac{\zeta}{k_{1}} \lambda_{*}\left(1-\lambda_{*}\right), \quad Z=\lambda_{*} \Delta .
$$

Furthermore, the derivatives of $\Delta$ and $Z$ evaluated at the FPs read

$$
\begin{aligned}
\partial_{m} \Delta & =\partial_{m} Z=0 & & \\
\partial_{a} \Delta & =-2 \frac{k_{a}}{k_{1}}, & \partial_{a} Z & =-\frac{k_{a}}{k_{1}}, \\
\partial_{\alpha} \Delta & =-2 \frac{k_{\alpha}}{k_{1}} \lambda_{*}, & \partial_{\alpha} Z & =\frac{k_{\alpha}}{k_{1}} \lambda_{*}\left(2-3 \lambda_{*}\right),
\end{aligned}
$$


The non-zero entries of the stability matrix defined as $H_{i j}=\left.\partial_{j} \beta_{i}\right|_{\mathrm{FP}}$ are given by

$$
\begin{aligned}
H_{m n} & =\frac{c_{G}}{2 k_{1}} \frac{\lambda_{*}}{\Delta} \delta_{m n}, \quad H_{a b}=\frac{c_{G}}{2 k_{1}} \frac{1-\lambda_{*}}{\Delta} \delta_{a b}, \\
H_{\alpha \beta} & =-\frac{c_{G}}{2 k_{1}} \frac{\lambda_{*}\left(1-\lambda_{*}\right)}{\Delta^{2}}\left(\Delta \delta_{\alpha \beta}-\frac{k_{\beta}}{k_{1}} \lambda_{*}\left(2-\lambda_{*}\right)\right), \\
H_{\alpha a} & =-\frac{c_{G}}{2 k_{1}} \frac{\lambda_{*}\left(1-\lambda_{*}\right)}{\Delta^{2}} \frac{k_{a}}{k_{1}}\left(1-2 \lambda_{*}\right) .
\end{aligned}
$$

Note that $H_{\alpha a}=0$, so that the stability matrix is not a symmetric one. Clearly the part of this matrix corresponding to the $n_{1}$ values $\lambda_{m}=0$ decouples. For the eigenvalue problem for the rest of the stability matrix we clearly obtain a matrix of the form

$$
\left(\begin{array}{ll}
A & \mathbf{0} \\
B & C
\end{array}\right)=\left(\begin{array}{ll}
\nVdash & \mathbf{0} \\
\mathbf{0} & C
\end{array}\right)\left(\begin{array}{cc}
A & \mathbf{0} \\
C^{-1} B & \nVdash
\end{array}\right) .
$$

Specifically, we have the matrix elements $A_{a b}=H_{a b}-E \delta_{a b}$ already in diagonal form with $E$ appearing in the left hand side of (4.15) being an eigenvalue of the stability matrix. The matrix $C_{\alpha \beta}=H_{\alpha \beta}-E \delta_{\alpha \beta}$ is of the form $c_{1} \delta_{\alpha \beta}-c_{2} k_{\beta}$ and as a result its determinant is equal to $c_{1}^{n_{3}-1}\left(c_{1}-\zeta c_{2}\right)$. Furthermore, the second matrix in the right hand side of (4.15) is triangular. The vanishing of the determinant of the matrix in (4.15) gives the eigenvalues of the stability matrix, as well as the corresponding degeneracies

$$
\begin{aligned}
\text { Deg } & =n_{1}: & H_{0} & =\frac{c_{G}}{2 k_{1}} \frac{\lambda_{*}}{\Delta}, \\
\operatorname{Deg} & =n_{2}: & H_{1} & =\frac{c_{G}}{2 k_{1}} \frac{1-\lambda_{*}}{\Delta}, \\
\operatorname{Deg} & =n_{3}-1: & H_{*} & =-\frac{c_{G}}{2 k_{1}} \frac{\lambda_{*}\left(1-\lambda_{*}\right)}{\Delta}, \\
\operatorname{Deg} & =1: & H_{1 *} & =\frac{c_{G}}{2 k_{1}} \frac{\zeta}{k_{1}} \frac{\lambda_{*}^{2}\left(1-\lambda_{*}\right)}{\Delta^{2}} .
\end{aligned}
$$

These eigenvalues and their degeneracies will be instrumental below in determining the physical IR stable fixed points of the RG flow.

\subsubsection{IR stable fixed points}

It turns out that in order to have a Euclidean signature for the metric, the constant $\Delta$ should be positive leading to the following condition

$$
0<\lambda_{*}<1 \quad \Longleftrightarrow \quad \rho<k_{1}<\rho+\zeta
$$

As mentioned before we will only consider cases with $n_{3} \geqslant 1$. In addition, physical fixed points cannot have $\lambda_{1}=1$ since the $\sigma$-model action (3.7) is in that case singular.

We are primarily interested in identifying the physical IR stable points, that is the ones which have all eigenvalues of the stability matrix positive. The condition (4.17) guaranties that $H_{0}>0$ and $H_{1}>0$. However, the third eigenvalue in (4.16) is negative, i.e. $H_{*}<0$ 
and therefore we should necessarily have $n_{3}=1$ so that this eigenvalue is non-existing. Finally, the positivity of the last eigenvalue $H_{1 *}$ is also guaranteed by (4.17).

The condition $n_{3}=1$ means that there is a single coupling that is not zero or one. Keeping in mind that $\lambda_{1} \neq 1$, there are two cases. In the first one let $\lambda_{1}=0$ and a single $\lambda_{a}=\lambda_{*}$. Then also $\zeta=k_{a}$. The condition (4.17) implies that $\rho<k_{1}<\rho+k_{a}$. The second case is when $\lambda_{1}=\lambda_{*}$. In this case $\lambda_{1}=1-\rho / k_{1}$ which holds automatically since according to (4.17). Also, in this case $\zeta=k_{1}$. We conclude that there is a multitude of fixed points the number of which depends on the relative ordering of the levels $k_{i}$. It would be certainly interesting to study the structure and properties of the corresponding CFTs.

\section{The non-Abelian T-duality limit}

When some of the $\lambda$ 's approach unity then we get a singularity in the manifold. Then a zoom in procedure maybe applied as in [6]. We will not discuss this for the most general action (2.13) but for (3.7) corresponding to integrable models.

In that case near $\lambda_{11}=1$ or near $\lambda_{n n}=1$ we get a singularity in the manifold. However, one may zoom in by taking simultaneously the large $k_{1}$ and $k_{n}$ limits. Then we expand for $k_{1}, k_{n} \gg 1$ as follows

$$
\begin{aligned}
& \lambda_{11}=1-\frac{1}{2 \zeta_{1} k_{1}}+\cdots, \quad g_{1}=\mathbb{I}+\frac{i}{2 \zeta_{1}} \frac{\left(v_{1}\right)_{a} t^{a}}{k_{1}}+\cdots, \\
& \lambda_{n n}=1-\frac{1}{2 \zeta_{n} k_{n}}+\cdots, \quad g_{n}=\mathbb{I}+\frac{i}{2 \zeta_{n}} \frac{\left(v_{n}\right)_{a} t^{a}}{k_{n}}+\cdots,
\end{aligned}
$$

where $\zeta_{1}$ and $\zeta_{n}$ are new coupling parameters. This leads to the following expressions for the currents and the operators $D_{i}$

$$
\begin{aligned}
& J_{1 \pm}^{a}=\frac{1}{2 \zeta_{1}} \frac{\partial_{ \pm} v_{1}^{a}}{k_{1}}+\cdots, \quad\left(D_{1}\right)_{a b}=\delta_{a b}+\frac{1}{2 \zeta_{1}} \frac{\left(f_{1}\right)_{a b}}{k_{1}}+\cdots, \quad\left(f_{1}\right)_{a b}=f_{a b c} v_{1}^{c}, \\
& J_{n \pm}^{a}=\frac{1}{2 \zeta_{n}} \frac{\partial_{ \pm} v_{n}^{a}}{k_{n}}+\cdots, \quad\left(D_{n}\right)_{a b}=\delta_{a b}+\frac{1}{2 \zeta_{n}} \frac{\left(f_{n}\right)_{a b}}{k_{n}}+\cdots, \quad\left(f_{n}\right)_{a b}=f_{a b c} v_{n}^{c},
\end{aligned}
$$

In this limit the action (3.7) becomes

$$
\begin{aligned}
S= & \sum_{i=2}^{n-1} S_{k_{i}}\left(g_{i}\right) \\
& +\frac{1}{2 \pi \zeta_{1}} \int d^{2} \sigma \partial_{+} v_{1}^{a}\left(\nVdash+f_{1}\right)_{a b}^{-1} \partial_{-} v_{1}^{b}+\frac{1}{2 \pi \zeta_{n}} \int d^{2} \sigma \partial_{+} v_{n}^{a}\left(\nVdash+f_{n}\right)_{a b}^{-1} \partial_{-} v_{n}^{b} \\
& +\frac{1}{\pi} \sum_{i=2}^{n-1} k_{i} \hat{\lambda}_{i 1} \int d^{2} \sigma J_{i+}\left(\nVdash+f_{1}\right)^{-1} \partial_{-} v_{1}+\frac{1}{\pi} \sum_{i=2}^{n-1} k_{i} \hat{\lambda}_{n i} \int d^{2} \sigma \partial_{+} v_{n}\left(\nVdash+f_{n}\right)^{-1} J_{i-} \\
& +\frac{1}{\pi} \sum_{i=2}^{n-1} k_{i} \hat{\lambda}_{i 1} \hat{\lambda}_{n i} \int d^{2} \sigma \partial_{+} v_{n}\left(\nVdash+f_{n}\right)^{-1} D_{i}^{T}\left(\nVdash+f_{1}\right)^{-1} \partial_{-} v_{1} .
\end{aligned}
$$

Note that Euclidean signature imposes a constraint on the parameters

$$
\zeta_{1}>0, \quad \zeta_{n}>0, \quad \zeta_{1} \sum_{i=2}^{n-1} \hat{\lambda}_{i 1}^{2}<1, \quad \zeta_{n} \sum_{i=2}^{n-1} \hat{\lambda}_{n i}^{2}<1 .
$$


This $\sigma$-model represents the interaction of $(n-2)$ WZW models for a group $G$ with two nonAbelian T-duals of the PCM for the same group which in turn interact among themselves. We note that the $\sigma$-model (5.3) being the limit of (3.7) is itself integrable. The original action whose non-Abelian T-dual is (5.3) is that of interacting PCMs.

An interesting limit of (5.3) arises if we take the remaining levels $k_{i} \rightarrow \infty$ as

$$
\begin{array}{rlrl}
g_{i} & =\mathbb{I}+i \frac{x_{i}^{a} t_{a}}{\sqrt{k_{i}}}+\cdots, & \hat{\lambda}_{i 1} & =\frac{\zeta_{i 1}}{\sqrt{k_{i}}}, \quad \hat{\lambda}_{n i}=\frac{\zeta_{n i}}{\sqrt{k_{i}}}, \\
k_{i} \rightarrow \infty, & i & =2,3, \ldots, n-1 .
\end{array}
$$

Then (5.3) becomes

$$
\begin{aligned}
S= & \frac{1}{2 \pi} \sum_{i=2}^{n-1} \partial_{+} x_{i}^{a} \partial_{-} x_{i}^{a} \\
& +\frac{1}{2 \pi \zeta_{1}} \int d^{2} \sigma \partial_{+} v_{1}^{a}\left(\nVdash+f_{1}\right)_{a b}^{-1} \partial_{-} v_{1}^{b}+\frac{1}{2 \pi \zeta_{n}} \int d^{2} \sigma \partial_{+} v_{n}^{a}\left(\nVdash+f_{n}\right)_{a b}^{-1} \partial_{-} v_{n}^{b} \\
& +\frac{1}{\pi} \sum_{i=2}^{n-1} \zeta_{i 1} \int d^{2} \sigma \partial_{+} x_{i}\left(\nVdash+f_{1}\right)^{-1} \partial_{-} v_{1}+\frac{1}{\pi} \sum_{i=2}^{n-1} \zeta_{n i} \int d^{2} \sigma \partial_{+} v_{n}\left(\nVdash+f_{n}\right)^{-1} \partial_{-} x_{i} \\
& +\frac{1}{\pi} \sum_{i=2}^{n-1} \zeta_{i 1} \zeta_{n i} \int d^{2} \sigma \partial_{+} v_{n}\left(\nVdash+f_{n}\right)^{-1}\left(\nVdash+f_{1}\right)^{-1} \partial_{-} v_{1} .
\end{aligned}
$$

This represents the mutual interactions of $(n-2) \operatorname{dim} G$ free fields with the two non-Abelian T-duals of PCM for a group $G$ and is also integrable. We may clearly simply even further by consistently taking $\zeta_{i 1}=0$ or $\zeta_{n i}=0$.

\section{Discussion and future directions}

We have construct the all loop effective action of a general class of models whose UV Lagrangian is the sum of an arbitrary number $n$ of WZW models based on the same group $G$, but at different levels. Although the complete effective action can be quite involved, at the linear level the theory is driven away from the conformal point by operators bilinear in the WZW currents. These current bi-linears involve currents belonging to both the same and different CFTs. Hence we have self- as well as mutual interactions of current algebra theories in their most general form. In general our models depend on $n^{2}$ general coupling matrices. We considered a consistent truncation of our models that depends on $2(n-1)$ couplings and showed that the theory is classically integrable by finding the appropriate Lax connection. Turning on even one more coupling will generate other couplings through quantum corrections and the theory will most likely cease to be integrable. Subsequently, we proved that the theory possesses certain non-perturbative symmetries in the space of couplings $\hat{\lambda}_{i 1}$ and $\hat{\lambda} n i$ and calculated the exact in the deformation parameters $\beta$-functions of our models which can be cast in a particularly simple and compact form. This fact allowed us to fully determine and classify the fixed points of the RG flow in particular those that are IR stable. Last but not least we consider non-Abelian T-duality type limits in the case when the theory is integrable. 
A number of open questions remain to be addressed. Given that our models provide concrete realizations of integrable flows between exact CFTs it would be interesting to elucidate the nature and symmetries of the corresponding IR stable CFTs. Furthermore, one could exploit the aforementioned non-perturbative symmetries that our models enjoy to compute the all-loop anomalous dimensions of current operators, as well as that of primary operators in a similar manner to that in $[7,14,20-22,24]$. One could also calculate the exact in the deformation parameters $C$-function of the models presented here as was done in [24] for simpler cases. In that respect the general results in [24] should be a useful starting point.

Another direction would be to consider the case where more of the couplings are nonzero, as well as the case where each of the WZW models is based on a different group. It is notable that in the latter case all formulae of section 2 will still be valid after slight modifications. In addition, one could search for integrable deformations in these more general cases. Compared to the integrable models presented in this work, we expect an even richer structure of the RG equations to be unveiled. Recently, a class of integrable models consisting of $N$ coupled principal chiral models each with a WZW term and based on the same group $G$ was presented in [54]. The construction was based on an association of integrable field theories with affine Gaudin models having an arbitrary number of sites. It would be interesting to see if these models bear any relation to the ones constructed in this work (see also [10]) or to the models with $N$ sites firstly presented in [7].

Our approach is valid to all orders in the deformation parameters but only to leading order in the $1 / k$ expansion, i.e. for large levels of the WZW action. One may wonder of how corrections of order $1 / k^{2}$ or higher may be computed. Although constructing the $\frac{1}{k}$ corrections to the effective action of the present work seems a hard task, one may resort to the higher order derivative $\beta$-functions of the $\sigma$-model. This will be helpful in computing corrections to the $\beta$-functions of the deformation parameters using gravitational methods and also in discovering the corrected version of non-perturbative symmetries of the type (3.9). In addition, we may compute the subleading terms in the $1 / k$ expansion of the $\beta$-functions, as well as of the anomalous dimensions of operators using CFT techniques. Finally, one could try to embed our models to solutions of type-IIB or type-IIA supergravity (for $n=2,3$ and small rank semisimple groups) and/or construct the corresponding $\eta$ deformed integrable models.

\section{Acknowledgments}

The work of G.G. on this project has received funding from the Hellenic Foundation for Research and Innovation (HFRI) and the General Secretariat for Research and Technology (GSRT), under grant agreement No 15425. K.S. would like to thank the Theoretical Physics Department of CERN for hospitality and financial support during part of this research.

Open Access. This article is distributed under the terms of the Creative Commons Attribution License (CC-BY 4.0), which permits any use, distribution and reproduction in any medium, provided the original author(s) and source are credited. 


\section{References}

[1] J.M. Maldacena, The Large $N$ limit of superconformal field theories and supergravity, Int. J. Theor. Phys. 38 (1999) 1113 [hep-th/9711200] [INSPIRE].

[2] M. Staudacher, The Factorized S-matrix of CFT/AdS, JHEP 05 (2005) 054 [hep-th/0412188] [INSPIRE].

[3] J. Ambjørn, R.A. Janik and C. Kristjansen, Wrapping interactions and a new source of corrections to the spin-chain/string duality, Nucl. Phys. B 736 (2006) 288 [hep-th/0510171] [INSPIRE].

[4] N. Gromov, V. Kazakov and P. Vieira, Exact Spectrum of Anomalous Dimensions of Planar $N=4$ Supersymmetric Yang-Mills Theory, Phys. Rev. Lett. 103 (2009) 131601 [arXiv:0901.3753] [INSPIRE].

[5] N. Beisert et al., Review of AdS/CFT Integrability: An Overview, Lett. Math. Phys. 99 (2012) 3 [arXiv: 1012.3982] [INSPIRE].

[6] K. Sfetsos, Integrable interpolations: From exact CFTs to non-Abelian T-duals, Nucl. Phys. B 880 (2014) 225 [arXiv:1312.4560] [INSPIRE].

[7] G. Georgiou, K. Sfetsos and K. Siampos, Double and cyclic $\lambda$-deformations and their canonical equivalents, Phys. Lett. B 771 (2017) 576 [arXiv:1704.07834] [INSPIRE].

[8] G. Georgiou and K. Sfetsos, A new class of integrable deformations of CFTs, JHEP 03 (2017) 083 [arXiv:1612.05012] [INSPIRE].

[9] G. Georgiou and K. Sfetsos, Integrable flows between exact CFTs, JHEP 11 (2017) 078 [arXiv: 1707.05149] [INSPIRE].

[10] G. Georgiou and K. Sfetsos, Novel all loop actions of interacting CFTs: Construction, integrability and RG flows, Nucl. Phys. B 937 (2018) 371 [arXiv:1809.03522] [INSPIRE].

[11] G. Itsios, K. Sfetsos and K. Siampos, The all-loop non-Abelian Thirring model and its RG flow, Phys. Lett. B 733 (2014) 265 [arXiv:1404.3748] [INSPIRE].

[12] K. Sfetsos and K. Siampos, Gauged WZW-type theories and the all-loop anisotropic non-Abelian Thirring model, Nucl. Phys. B 885 (2014) 583 [arXiv:1405.7803] [InSPIRE].

[13] D. Kutasov, Duality Off the Critical Point in Two-dimensional Systems With Nonabelian Symmetries, Phys. Lett. B 233 (1989) 369 [InSPIRE].

[14] G. Georgiou, E. Sagkrioti, K. Sfetsos and K. Siampos, Quantum aspects of doubly deformed CFTs, Nucl. Phys. B 919 (2017) 504 [arXiv: 1703.00462] [InSPIRE].

[15] E. Sagkrioti, K. Sfetsos and K. Siampos, RG flows for $\lambda$-deformed CFTs, Nucl. Phys. B 930 (2018) 499 [arXiv: 1801.10174] [INSPIRE].

[16] D. Kutasov, String Theory and the Nonabelian Thirring Model, Phys. Lett. B 227 (1989) 68 [INSPIRE].

[17] B. Gerganov, A. LeClair and M. Moriconi, On the $\beta$-function for anisotropic current interactions in 2-D, Phys. Rev. Lett. 86 (2001) 4753 [hep-th/0011189] [InSPIRE].

[18] A. LeClair, Chiral stabilization of the renormalization group for flavor and color anisotropic current interactions, Phys. Lett. B 519 (2001) 183 [hep-th/0105092] [INSPIRE].

[19] C. Appadu and T.J. Hollowood, $\beta$-function of $k$ deformed $A d S_{5} \times S^{5}$ string theory, JHEP 11 (2015) 095 [arXiv: 1507.05420] [INSPIRE]. 
[20] G. Georgiou, K. Sfetsos and K. Siampos, All-loop anomalous dimensions in integrable $\lambda$-deformed $\sigma$-models, Nucl. Phys. B 901 (2015) 40 [arXiv:1509. 02946] [INSPIRE].

[21] G. Georgiou, K. Sfetsos and K. Siampos, All-loop correlators of integrable $\lambda$-deformed $\sigma$-models, Nucl. Phys. B 909 (2016) 360 [arXiv:1604.08212] [INSPIRE].

[22] G. Georgiou, K. Sfetsos and K. Siampos, $\lambda$-Deformations of left-right asymmetric CFTs, Nucl. Phys. B 914 (2017) 623 [arXiv:1610.05314] [INSPIRE].

[23] A.B. Zamolodchikov, Irreversibility of the Flux of the Renormalization Group in a 2D Field Theory, JETP Lett. 43 (1986) 730 [INSPIRE].

[24] G. Georgiou, P. Panopoulos, E. Sagkrioti, K. Sfetsos and K. Siampos, The exact C-function in integrable $\lambda$-deformed theories, Phys. Lett. B 782 (2018) 613 [arXiv:1805.03731] [INSPIRE].

[25] E. Sagkrioti, K. Sfetsos and K. Siampos, Weyl anomaly and the $C$-function in $\lambda$-deformed CFTs, Nucl. Phys. B 938 (2019) 426 [arXiv:1810.04189] [InSPIRE].

[26] T.J. Hollowood, J.L. Miramontes and D.M. Schmidtt, Integrable Deformations of Strings on Symmetric Spaces, JHEP 11 (2014) 009 [arXiv:1407.2840] [INSPIRE].

[27] T.J. Hollowood, J.L. Miramontes and D.M. Schmidtt, An Integrable Deformation of the $A d S_{5} \times S^{5}$ Superstring, J. Phys. A 47 (2014) 495402 [arXiv:1409.1538] [InSPIRE].

[28] K. Sfetsos and K. Siampos, Integrable deformations of the $G_{k_{1}} \times G_{k_{2}} / G_{k_{1}+k_{2}}$ coset CFTs, Nucl. Phys. B 927 (2018) 124 [arXiv:1710.02515] [inSPIRE].

[29] J. Balog, P. Forgacs, Z. Horvath and L. Palla, A New family of SU(2) symmetric integrable $\sigma$-models, Phys. Lett. B 324 (1994) 403 [hep-th/9307030] [INSPIRE].

[30] K. Sfetsos and K. Siampos, The anisotropic $\lambda$-deformed $\mathrm{SU}(2)$ model is integrable, Phys. Lett. B 743 (2015) 160 [arXiv:1412.5181] [INSPIRE].

[31] K. Sfetsos, K. Siampos and D.C. Thompson, Generalised integrable $\lambda$ - and $\eta$-deformations and their relation, Nucl. Phys. B 899 (2015) 489 [arXiv: 1506.05784] [INSPIRE].

[32] K. Sfetsos and D.C. Thompson, Spacetimes for $\lambda$-deformations, JHEP 12 (2014) 164 [arXiv: 1410.1886] [INSPIRE].

[33] S. Demulder, K. Sfetsos and D.C. Thompson, Integrable $\lambda$-deformations: Squashing Coset CFTs and $A d S_{5} \times S^{5}$, JHEP 07 (2015) 019 [arXiv: 1504.02781] [INSPIRE].

[34] R. Borsato, A.A. Tseytlin and L. Wulff, Supergravity background of $\lambda$-deformed model for $A d S_{2} \times S^{2}$ supercoset, Nucl. Phys. B 905 (2016) 264 [arXiv:1601.08192] [INSPIRE].

[35] Y. Chervonyi and O. Lunin, Supergravity background of the $\lambda$-deformed $A d S_{3} \times S^{3}$ supercoset, Nucl. Phys. B 910 (2016) 685 [arXiv:1606.00394] [INSPIRE].

[36] B. Vicedo, Deformed integrable $\sigma$-models, classical R-matrices and classical exchange algebra on Drinfel'd doubles, J. Phys. A 48 (2015) 355203 [arXiv:1504.06303] [INSPIRE].

[37] B. Hoare and A.A. Tseytlin, On integrable deformations of superstring $\sigma$-models related to $A d S_{n} \times S^{n}$ supercosets, Nucl. Phys. B 897 (2015) 448 [arXiv: 1504.07213] [INSPIRE].

[38] C. Klimčík, $\eta$ and $\lambda$ deformations as $\mathcal{E}$-models, Nucl. Phys. B 900 (2015) 259 [arXiv: 1508.05832] [INSPIRE].

[39] C. Klimčík, Poisson-Lie T-duals of the bi-Yang-Baxter models, Phys. Lett. B 760 (2016) 345 [arXiv: 1606.03016] [INSPIRE]. 
[40] B. Hoare and F.K. Seibold, Poisson-Lie duals of the $\eta$-deformed $A d S_{2} \times S^{2} \times T^{6}$ superstring, JHEP 08 (2018) 107 [arXiv: 1807.04608] [INSPIRE].

[41] C. Klimčík and P. Ševera, Dual nonAbelian duality and the Drinfeld double, Phys. Lett. B 351 (1995) 455 [hep-th/9502122] [INSPIRE].

[42] K. Sfetsos, Duality invariant class of two-dimensional field theories, Nucl. Phys. B 561 (1999) 316 [hep-th/9904188] [INSPIRE].

[43] C. Klimčík, Yang-Baxter $\sigma$-models and dS/AdS T duality, JHEP 12 (2002) 051 [hep-th/0210095] [INSPIRE].

[44] C. Klimčík, On integrability of the Yang-Baxter $\sigma$-model, J. Math. Phys. 50 (2009) 043508 [arXiv:0802.3518] [INSPIRE].

[45] C. Klimčík, Integrability of the bi-Yang-Baxter $\sigma$-model, Lett. Math. Phys. 104 (2014) 1095 [arXiv: 1402.2105] [INSPIRE].

[46] F. Delduc, M. Magro and B. Vicedo, On classical q-deformations of integrable $\sigma$-models, JHEP 11 (2013) 192 [arXiv:1308.3581] [INSPIRE].

[47] F. Delduc, M. Magro and B. Vicedo, An integrable deformation of the $A d S_{5} \times S^{5}$ superstring action, Phys. Rev. Lett. 112 (2014) 051601 [arXiv:1309.5850] [INSPIRE].

[48] G. Arutyunov, R. Borsato and S. Frolov, S-matrix for strings on $\eta$-deformed $A d S_{5} \times S_{5}$, JHEP 04 (2014) 002 [arXiv: 1312.3542] [INSPIRE].

[49] O. Lunin and W. Tian, Scalar fields on $\lambda$-deformed cosets, Nucl. Phys. B 938 (2019) 671 [arXiv: 1808.02971] [INSPIRE].

[50] D.M. Schmidtt, Integrable Lambda Models And Chern-Simons Theories, JHEP 05 (2017) 012 [arXiv: 1701.04138] [INSPIRE].

[51] D.M. Schmidtt, Lambda Models From Chern-Simons Theories, JHEP 11 (2018) 111 [arXiv: 1808.05994] [INSPIRE].

[52] S. Driezen, A. Sevrin and D.C. Thompson, D-branes in $\lambda$-deformations, JHEP 09 (2018) 015 [arXiv: 1806.10712] [INSPIRE].

[53] P. Bowcock, Canonical Quantization of the Gauged Wess-Zumino Model, Nucl. Phys. B 316 (1989) 80 [INSPIRE].

[54] F. Delduc, S. Lacroix, M. Magro and B. Vicedo, Integrable Coupled $\sigma$ Models, Phys. Rev. Lett. 122 (2019) 041601 [arXiv: 1811.12316] [INSPIRE]. 